\title{
Richard Saint-Gelais, Fictions transfuges, La trans- fictionnalité et ses enjeux
}

\section{Mariangela Napolitano}

\section{(2) OpenEdition}

1 Journals

\section{Edizione digitale}

URL: http://journals.openedition.org/studifrancesi/3920

DOI: $10.4000 /$ studifrancesi.3920

ISSN: 2421-5856

\section{Editore}

Rosenberg \& Sellier

\section{Edizione cartacea}

Data di pubblicazione: 1 décembre 2012

Paginazione: 626-627

ISSN: 0039-2944

\section{Notizia bibliografica digitale}

Mariangela Napolitano, «Richard Saint-Gelais, Fictions transfuges, La trans-fictionnalité et ses enjeux»,

Studi Francesi [Online], 168 (LVI | III) | 2012, online dal 30 novembre 2015, consultato il 07 mars 2021. URL: http://journals.openedition.org/studifrancesi/3920 ; DOI: https://doi.org/10.4000/studifrancesi. 3920

Questo documento è stato generato automaticamente il 7 mars 2021.

\section{(c) (i) $\odot$}

Studi Francesi è distribuita con Licenza Creative Commons Attribuzione - Non commerciale - Non opere derivate 4.0 Internazionale. 


\title{
Richard Saint-Gelais, Fictions transfuges, La trans-fictionnalité et ses enjeux
}

\author{
Mariangela Napolitano
}

\section{NOTIZIA}

RICHARD SAINT-GELAIS, Fictions transfuges, La trans-fictionnalité et ses enjeux, Paris, Éditions du Seuil, 2011, pp. 602.

1 Richard SAINT-GeLAIS, professore di letteratura del xx secolo all'Università di Laval in Québec, e specialista del nouveau roman e della cosiddetta paraletteratura, ha impiegato diversi anni per la composizione di questo suo prezioso e interessante saggio. Prezioso e interessante anche il fulcro attorno al quale il nostro autore fa girare tutti gli ingranaggi della sua macchina saggistica: la transfictionnalité, termine che allude a una sorta di finzione nella finzione. Sembra quasi un gioco, un gioco di curiosità per l'esattezza, ben diffuso tra autori e lettori di un testo: chi non si è mai soffermato a immaginare tutti i fili della storia che lo scrittore decideva di non seguire, o le mille altre facce di un personaggio che per questioni narrative finivano col restare in ombra? Richard Saint-Gelais in un'intervista afferma che ciò che gli ha fornito il "la" per cominciare questo suo studio è stata la fantascienza, e in particolar modo Star Trek che, in quanto saga più che celebre, ha fornito nel corso degli anni una serie tentacolare di rivisitazioni, non soltanto dal punto di vista dell'impianto narrativo, ma anche dei mezzi di diffusione, spaziando tra letteratura, cinema, televisione.

2 L'A. prende in considerazione soprattutto il modello seriale per eccellenza, Sherlock Holmes, ma anche i protagonisti di opere classiche di letteratura, come Madame Bovary o Don Quichotte. Questo gli ha permesso di comprendere che la transfictionnalité nasce lì dove subentra un rifiuto verso la corrispondenza univoca tra una finzione e un testo come unica possibilità narrativa. Un confine questo talmente labile che viene infranto 
già dal lettore stesso di un'opera, prima ancora che dall'autore che si accinge ad apportare le modifiche volute.

Richard Saint-Gelais cerca di spiegare innanzitutto cosa sia esattamente la transfictionnalité nei primi due dei nove capitoli in cui è suddivisa la sua opera, «Contours» e «La fiction à travers l'intertexte»: potremmo definirlo quasi un fenomeno, più che una tecnica, riscontrabile quando due o più testi condividono gli stessi elementi della finzione, dove elemento sta a indicare tanto un personaggio, quanto un luogo o, ancora, un avvenimento. Ora, si tratti di un romanzo, un film, un fumetto, un graphic novel, una serie televisiva, il meccanismo non cambia, anzi la scelta diversa del medium non fa che amplificare il fenomeno. Ciò che rende tutto questo possibile è l'incompletezza che sta alla base del mondo della finzione, distante da quello del reale ma anche da quello del possibile. Ed è proprio questa incompletezza che, facendoci ignorare gran parte delle informazioni possibili, fa scattare la molla del gioco della finzione nella finzione. Il resto dello studio saggistico è un proseguire per tappe, o meglio per capitoli, attraverso tutte le possibili forme e manifestazioni della transfictionnalité.

4 Nel terzo capitolo, «Expansions», vengono passate in rassegna tutte le forme di transfictionnalité che muovono da un testo ben definito di partenza. Potremmo considerarli quasi degli ampliamenti, dai semplici sequels e prequels, cioè prolungamenti e "prologhi" della storia, passando per le interpolazioni, cioè ricondurre, anticipandola, la storia a due punti diversi ed esatti della narrazione, per le espansioni parallele, virtuali e paradossali, e le varie forme di serialità.

5 Nel quarto capitolo, «Versions», Saint-Gelais ci mostra invece come la transfictionnalité non adoperi soltanto le espansioni narrative, ma si manifesti anche sotto forma di versioni alternative della stessa opera, che siano reinterpretazioni, correzioni, decentramenti, o accentramenti, e così via.

6 Il quinto capitolo, «Croisements et annexions», esplora la possibilità in cui la diegesi preesistente, alla quale la transfictionnalité si aggrappa, non sia una bensì due o più, vale a dire due o più finzioni che prima di allora il lettore aveva considerato come indipendenti e che ora ritrova invece collegate. Esempio lampante potrebbe essere l'incontro tra Sherlock Holmes e Dracula in un'opera di Loren D. Estleman.

7 Il sesto capitolo, «Captures», studia quest'acrobazia molto rara da riscontrare in cui non viene stabilita una relazione tra testo e testo, quanto piuttosto tra una finzione e un testo, fenomeno spesso autografo, cioè realizzato dall'autore stesso delle opere di partenza.

8 Nel settimo capitolo, «Systèmes», trova spazio una sorta di cartografia del sistema di transfictionnalité e dei suoi legami, potremmo dire, transitivi, facendo una dovuta distinzione tra testi autografi e allografi.

9 L'ottavo capitolo, «Le stade médiatique de la fiction», indaga invece su come la cultura mediatica non abbia fatto altro che amplificare il fenomeno della trans- fictionnalité portando a dei casi che Saint-Gelais definisce di fan fiction.

10 L'ultimo capitolo, "Critique et transfictionnalité», mostra appunto il legame tra il fenomeno narrativo e la critica, come cioè un testo transfictionnel possa assumere in alcuni casi una funzione critica rispetto al testo di partenza. Procede quindi con l'indagare innanzitutto sulla critica della transfictionnalité per poi arrivare al suo esatto 
inverso, soffermandosi sulla varietà più facilmente identificabile di tale fenomeno, l'holmésologie. 